\title{
Statistical Modeling and Analysis of Wide Binary Star Systems
}

\author{
K.B. Johnston ${ }^{1}$, T.D. Oswalt ${ }^{1}$, and D. Valls-Gabaud ${ }^{2}$ \\ ${ }^{1}$ Dept. of Physics and Astronomy, Florida Institute of Technology \\ 150 West University Blvd., Melbourne, Fl 32901 \\ email: kyjohnst@fit.edu and toswalt@fit.edu \\ ${ }^{2}$ Laboratoire d'Astrophysique UMR CNRS 5572, Observatoire Midi-Pyre'ne'es \\ 14 Avenue Edouard Belin, F-31400 Toulouse, France. \\ email:David.Valls-Gabaud@obspm.fr
}

\begin{abstract}
Post-main-sequence (MS) mass loss causes orbital separation amplification in fragile (i.e. common proper motion) binary star systems. Components typically have separations around $\sim 1000$ AU. Such wide pairs experience negligible tidal interactions and mass transfer between companions; thus they evolve as two separate but coeval stars. In this paper we compute the rate of mass loss during the components' lifetimes and attempt to model how it will statistically distort a frequency distribution of fragile binary separations. Understanding this process provides a robust test of current theories of stellar evolution and sets constraints on the dynamics of the Galactic disk.
\end{abstract}

Keywords. binaries: general - stars: statistics

\section{Observation Data and Modeled Data}

A statistically significant frequency distribution of separations for each class of wide binary (MS+MS and WD+MS) can be obtained by sampling a large survey. The Luyten Double Star Catalog (Luyten 1969, 1974, 1979) is a large well-understood sample upon which to initially test our orbit evolution models. One of the major goals of this project is to evolve a theoretical population of stars while keeping track of its mass, luminosity, and temperature. We have chosen to employ the Single Star Evolution (SSE) program (Hurley, Pols \& Tout 2000; or HPT) to solve our evolution problem. We used SSE to produce a large population of synthetic evolving wide binaries. Following Pringle (1985), we can show that for non-conservative mass loss, binary period and separation increase.

\section{Results}

Figure 1 (top) displays the separation distribution for MS+MS pairs created by our models. Separation is given as $\log a$ in AU. The sharp observed limits are caused by our assumption of an Öpik distribution with upper and lower separation being limits typical of common proper motion binaries. Figure 1 (bottom) shows the expected general increase of separations imposed by post-MS mass loss. Note that mass loss has depleted the number of close binaries and increased the higher-separation binaries.

Figure 2 (top and bottom) shows comparisons between the translated model and the observed distributions (Luyten Sample). Curiously, the average angular separation of the Luyten sample has decreased from $63^{\prime \prime} .2$ to $52^{\prime \prime} .9$. This may be a selection effect resulting from closer and dimmer MS+MS pairs not being resolvable (i.e., not detectable).

Model separations were transformed into 'observable' angular separations using a log 


\section{Histogram of Separation Before}

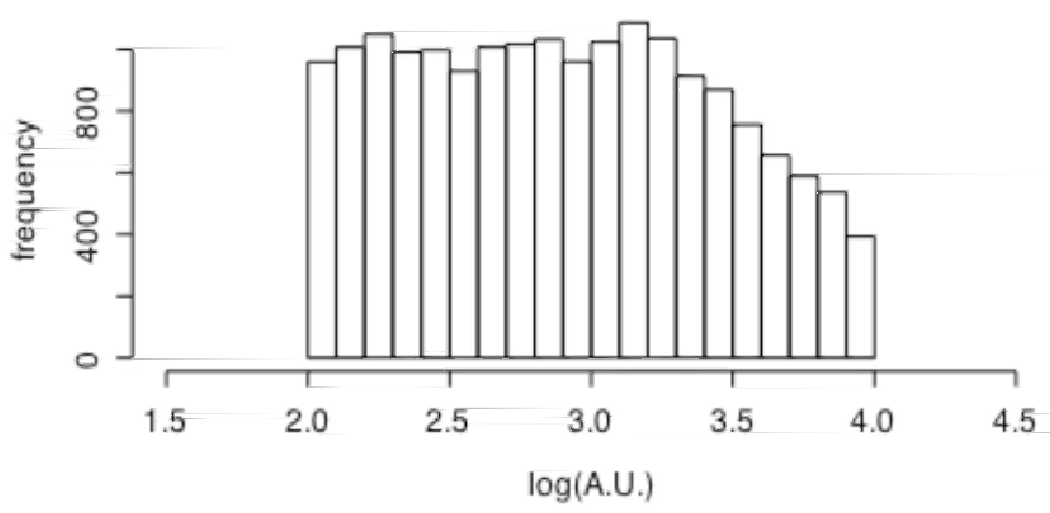

Histogram of Separation After

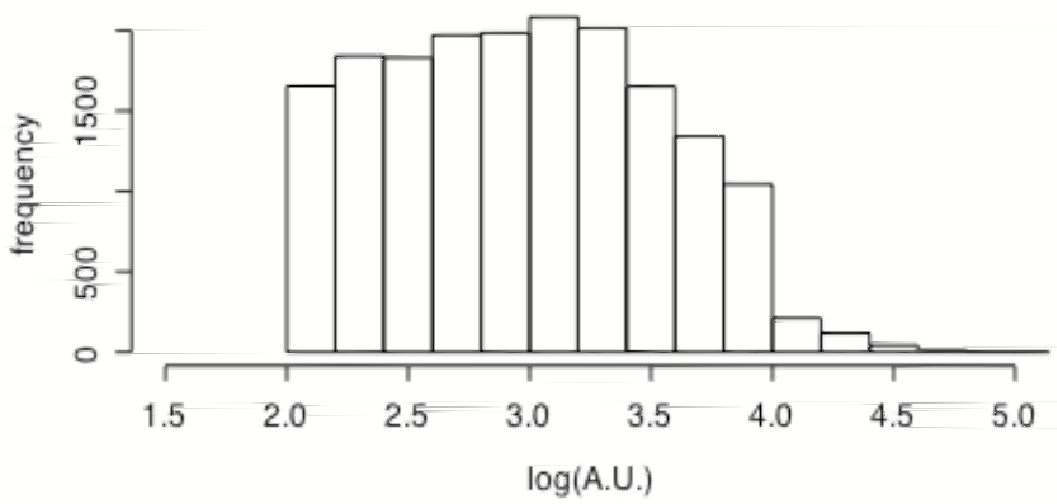

Figure 1. Histogram of the Distribution of Separations

Table 1. Statistical Characteristics of Model Distributions, Before and After

\begin{tabular}{|c|c|c|c|c|c|c|c|}
\hline Units & & Average & StdDev & Skew & Kurtosis & Min & $\operatorname{Max}$ \\
\hline Before & $\log a$ & 2.906 & 0.536 & 0.124 & -1.048 & 2.000 & 4.000 \\
\hline Before & $\log P(\mathrm{Myr}) .$. & -1.594 & 0.826 & 0.109 & -0.914 & -3.954 & 0.407 \\
\hline & & & & 2 & & 2.000 & 5.048 \\
\hline After & $\log P(\mathrm{Myr})$ & -1.464 & 0.833 & 0.138 & -0.887 & -3.160 & 0.966 \\
\hline
\end{tabular}

distance model, where distance is a random function based on $3 x+1 ; d=10^{3 x+1}$ where $0<x<1$ and $x$ is a random number with uniform probability distribution. Identical histogram bin sizes were used for both observed and computed models. Because our sample sizes differ, we chose to convert our histogram of population size into percentages as seen in Figure 2, top and bottom. We are currently attempting to understand several aspects of this comparison: 
Observed Luyten Data vs. Theoretical Model from Double Star MS+MS Sample

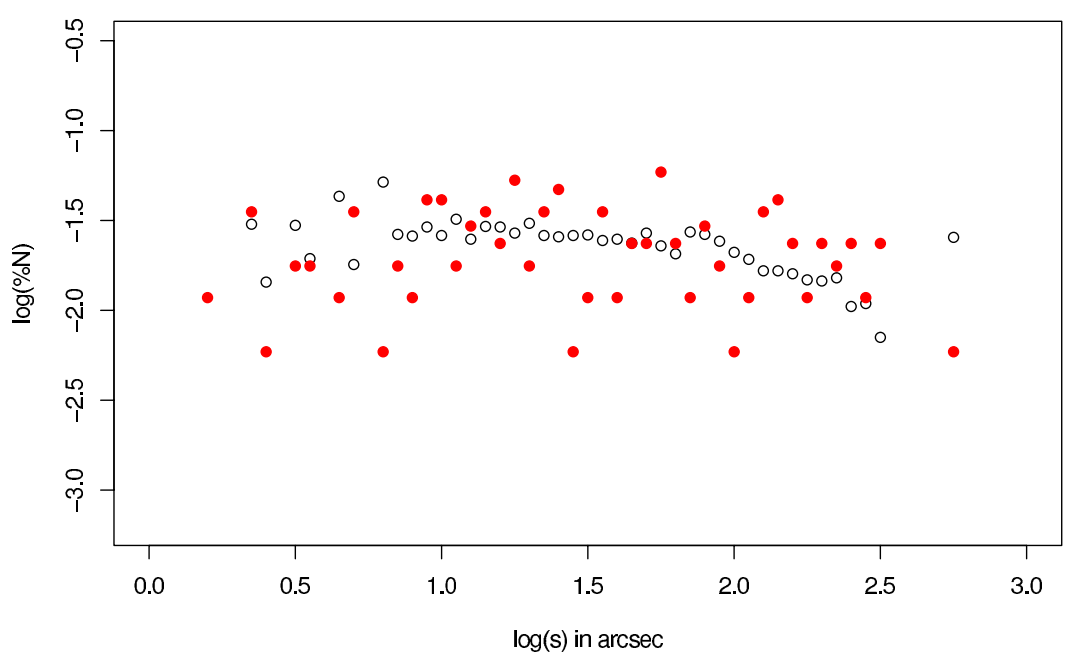

Observed Luyten Data vs. Theoretical Model from Double Star WD+MS Sample

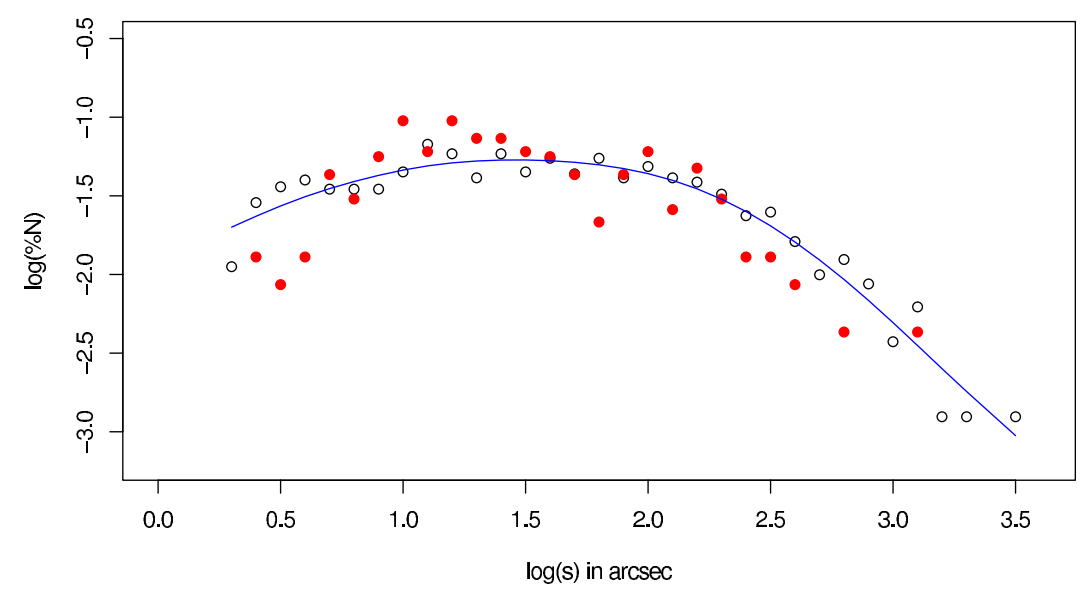

Figure 2. Log-Log Plot of the $\log (\% N)$ vs. Angular Separation. Observed: Red (Filled) and Model: Open. The Line is a Bi-Cubic Spline of the WD+MS Model Data

(1) The peakedness of the model is not as great as the peakedness of the observed sample. The density of binaries with small angular separations could have been underobserved by Luyten. Alternatively, our model may predict too many close binaries and our minimum angular separation limit might not realistic.

(2) The scatter in the observed data is $1 \sigma$ greater than the modeled data. With a larger sample the observed data scatter should decrease. 
Table 2. Statistical Characteristics of Evolved Distributions

\begin{tabular}{|c|c|c|c|c|c|c|}
\hline & Average & StdDev & Skew & Kurtosis & Min & $\operatorname{Max}$ \\
\hline LI & 47 & 0.042 & -0.380 & -0.581 & -2.230 & -1.23 \\
\hline $\mathrm{M}$ & -1.650 & .025 & -0.830 & 66 & -2.150 & -1.129 \\
\hline Residu & -0.097 & 0.017 & 0.450 & -2.257 & -0.080 & -0.102 \\
\hline LDS WD+MS log & -1.747 & 0.050 & -0.255 & -0.702 & -2.365 & -1.110 \\
\hline Model WD+MS log $\% 1$ & -1.676 & 0.089 & -1.483 & 1.296 & -2.905 & -1.172 \\
\hline Residual WD+Ms & 0.056 & 0.054 & 0.064 & -0.273 & -0.325 & 0.622 \\
\hline
\end{tabular}

\section{Future Work}

Completion of the project will require a more realistic distance model. We will attempt to apply a Monte-Carlo generated distance probability distribution for stellar population models. As mentioned above, we also plan to improve our observational sample by using the SDSS DR4 survey in conjunction with the USNO proper motion database. We also plan several related investigations: Markov-Chain modeling of future expectations, improved range of stellar masses, improved orbital mechanical evolution code, and the inclusion of large Jupiter-like planetary bodies as companions to binaries.

\section{Acknowledgements}

The authors would like to thank Rob Wilkos for his help in plotting and running some of the Double Star models. All graphs in this paper have been made with the R-project program. We would also like to thank Drs. Matt Wood and Stéphane Vennes for their support and advice. This project was supported in part by the NASA grant Y701296 (T.D.O.) and NSF grant AST 02-06115 (T.D.O.).

\section{References}

Hurley, J., Pols, O., \& Tout, C., 2000, MNRAS, 315, 543-569

Luyten, W.J., 1969, Proper Motion Survey with the 48-inch Schmidt Telescope, XXI Double Stars with Common Proper Motion, University of Minnesota

Pringle, J.E. 1985, in Interacting Binary Stars, ed. J.E. Pringle \& R.A. Wade (Cambridge: Cambridge Univ. Press), 1

Valls-Gabaud, D., 1988, ApESSS, 142, 289-304

Wasserman, I., 1988, Ap\&SSS, 142 267-276

Zinnecker, H., 1984, Ap\&SSS, 99, 41-70 